\title{
Histopathological Findings in an Autopsy of Covid-19 Positive Young Male
}

\author{
Sonia Chhabra ${ }^{1}$, Niti Dalal ${ }^{2 *}$, Renuka Verma ${ }^{3}$, Jyoti Dahiya ${ }^{4}$ and Sunita Singh ${ }^{5}$ \\ ${ }^{1}$ Professor, Department of Pathology, PGIMS, India \\ ${ }^{2}$ Senior Resident, Department of Pathology, PGIMS, India \\ ${ }^{3}$ Associate Professor, Department of Pathology, PGIMS, India \\ ${ }^{4}$ Junior Resident, Department of Pathology, PGIMS, India \\ ${ }^{5}$ Senior Professor \& Head, Department of Pathology, PGIMS, India
}

\begin{abstract}
First confirmed case of novel coronavirus was identified in December 2019 in Wuhan, China and designated as SARS coronavirus-2 (SARS-CoV-2). Afterwards, it spreads very rapidly all over the world. World Health Organization declared it as pandemic on $11^{\text {th }}$ march, 2020. The symptoms range from mild to severe acute respiratory distress syndrome leading to hospitalization and eventually, death of the patient. Lung is the predominant organ which is severely affected. Here, we present an autopsy finding in heart and lungs in a 22-year-old male who died with clinical diagnosis of pneumonia and ARDS in November 2020.
\end{abstract}

KEYWORDS: COVID 19; Autopsy; Heart; Lungs

ABBREVIATIONS: SARS-CoV-2: Severe Acute Respiratory Syndrome Coronavirus 2; MERS: Middle East Respiratory Syndrome; SARS: Severe Acute Respiratory Syndrome; ACE2: Angiotensin- Converting Enzyme 2; ARDS: Acute Respiratory Distress Syndrome

\section{INTRODUCTION}

Ever since it was first reported in China, all the countries around the world have faced outbreaks of COVID-19 and is a major cause of mortality worldwide. Italy was the first country to report a huge number of COVID-19 cases [1].

COVID-19 is caused by the severe acute respiratory syndrome coronavirus 2 (SARS-CoV-2) and is the seventh member of the coronavirus family [1]. Coronaviruses are single-stranded, enveloped RNA viruses that cause respiratory illnesses among humans and animals.

Disease cause by these viruses range from the common cold to severe acute respiratory syndrome (SARS) and Middle East respiratory syndrome (MERS) [2].Coronaviruses gain entry into cells via the angiotensin- converting enzyme 2 (ACE2), which is expressed in multiple tissue types including endothelium. The virus disproportionately affects the respiratory system and in elderly patients with comorbidities such as hypertension, diabetes, obesity, and cardiovascular disease. In respiratory system, it leads to diffuse alveolar damage, edema and a marked increase in lung weights at autopsy $[2,3]$.

SARS-CoV-2 infection also releases the significant amount of pro-inflammatory cytokines that aggravate interstitial pneumonia and acute respiratory distress syndrome (ARDS). This results into viral sepsis with prominent hypercoagulability and multiorgan dysfunction [3].

\begin{tabular}{|c|c|}
\hline \multirow{2}{*}{ 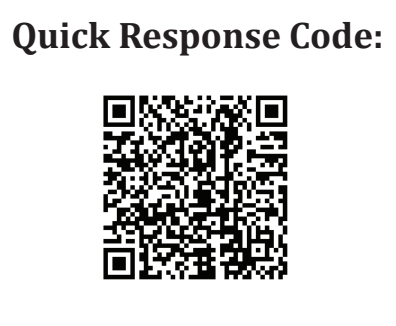 } & $\begin{array}{l}\text { Address for correspondence: Niti Dalal, Senior Resident, Department of Pathology, } \\
\text { PGIMS, India }\end{array}$ \\
\hline & $\begin{array}{l}\text { Received: July 19, } 2021 \quad \text { Published: August 13, } 2021 \\
\text { How to cite this article: Sonia C, Niti D, Renuka V, Jyoti D, Sunita S. Histopathological Findings } \\
\text { in an Autopsy of Covid-19 Positive Young Male. 2021- 3(4) OAJBS.ID.000315. DOI: 10.38125/ } \\
\text { OAJBS.000315 }\end{array}$ \\
\hline
\end{tabular}




\section{CASE REPORT}

A 22-year-old prisoner male presented with high grade fever and sore throat. RT-PCR for COVID-19 was found out to be positive. Following which, he was admitted to the hospital. He was given injection piperacillin, tazobactam, remdesivir, methyl prednisolone, thiamine and other supportive medication. After one week, patient developed respiratory distress. He was shifted to COVID ICU. There was constant dip in the SpO2, and patient was intubated. After some time, eventually his blood pressure was non recordable and pulse was not palpable and patient died. A clinical diagnosis was of COVID 19 positive with bilateral pneumonia and ARDS. A limited autopsy was performed after performing RTPCR again which came out to be negative. Heart and parts of both lungs were sent for histopathological examination, keeping in view the biosafety measures. Specimen was received in the Department of Pathology in $10 \%$ formalin. The jar was remained closed for another 10 days and afterwards, grossing was done with proper biosafety precautions. On gross examination, heart weighed $240 \mathrm{gm}$ and measured $11 \times 8.5 \times 5.5 \mathrm{~cm}$. Pieces of lung weighed $49 \mathrm{gm}$ and $39 \mathrm{gm}$, measured $8 \times 5.5 \times 3 \mathrm{~cm}$ and $9 \times 5 \times 3 \mathrm{~cm}$, respectively. Both the lung pieces were firm, heavy and showed liver like consistency. Microscopic section from various portion of heart show myocardium revealing diffuse by inflammatory cells comprising mainly lymphocytes and few eosinophils. Histopathological diagnosis of myocarditis was rendered. Sections from lung pieces revealed changes of pneumonia with mixed inflammatory infiltrate. A final diagnosis of myocarditis with pneumonia was made (Figure 1\&2).

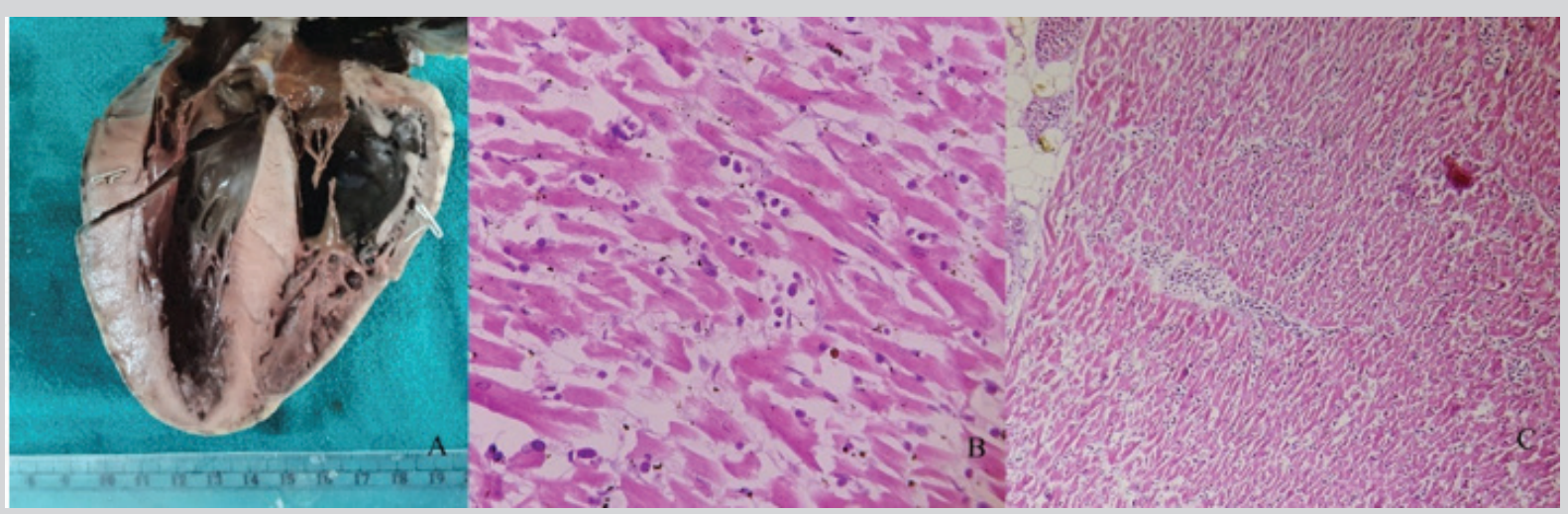

Figure 1: A: Gross appearance of heart; B, C: Inflammatory infiltrate seen in myocardium along with engorged vessels ( $H$ \& $E, 400 x, 100 x)$.

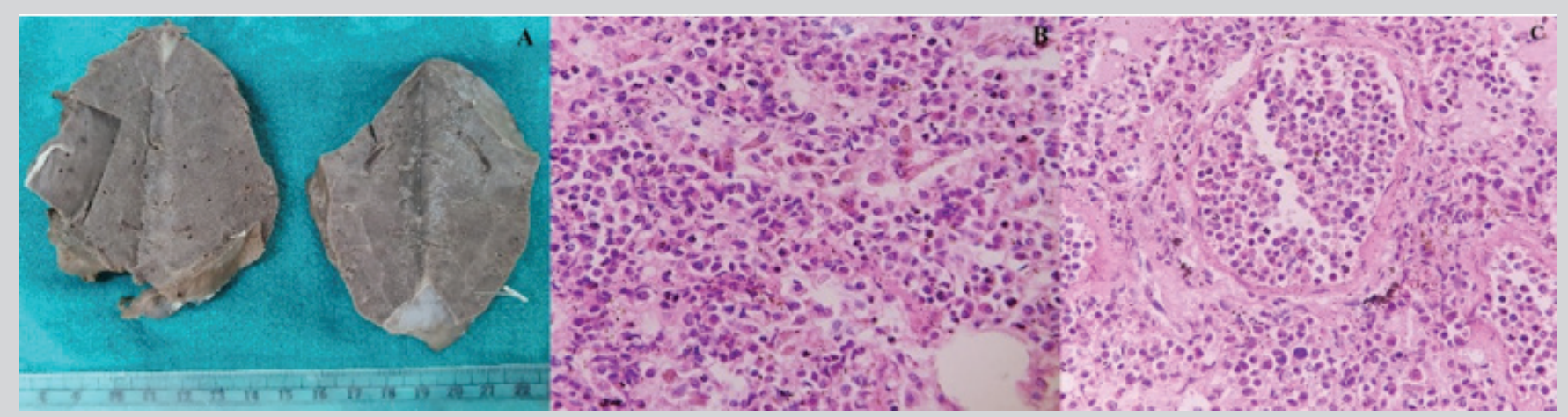

Figure 2: A: Gross appearance of lung showing liver like consistency; B, C: Changes of pneumonia along with engorged vessels (H \& $E, 400 x, 100 x)$.

\section{DISCUSSION}

Due to an increasing number of cases, COVID-19 has become a major challenge for all health care authorities [3]. Predominantly lung is the most common organ which is involved in patients with COVID-19 patients. Diffuse alveolar damage, hyaline membrane formation and pneumocyte atypical hyperplasia are frequent identified. Presence of microthrombi in vessels is consistent with coagulopathy, which appears to be common in patients with COVID-19 and should be one of the main targets of therapy. The clinical presentation ranged from asymptomatic, mild upper respiratory infection with fever and cough, to severe cases, with pneumonia, and progressing onto acute respiratory distress syndrome (ARDS). In severe cases, the main histological findings revealed exudative diffuse alveolar damage with massive capillary congestion, accompanied by microthrombi [1]. The histological features of cellular and interstitial damage in lungs were graded on a semiquantitative scale on the basis of the percentage of tissue involved: absent $(0 \%)$, rare $(<5 \%)$, focal $(5-25 \%)$, multifocal (26$50 \%$ ), plurifocal (51-75\%), or diffuse $(>75 \%)$ [1].

Elsoukkary et al. [2] studied 32 autopsies of patients who died from SARS-CoV-2 and having comorbidities, viz, hypertension, coronary artery disease, obesity, and diabetes. Lung findings are the most significant for the majority of the cases, while other organs (liver, kidney and lymph node) are also frequently involved having widespread microscopic thrombosis. Morphological findings in lung are variable ranging from few normal areas, patchy exudative hyaline membrane disease to organizing pneumonia. It is also believed that these patients have a propensity to develop microthrombi in the myocardial vessels and also causes myocarditis. In view of widespread involvement of other organs like liver, kidney 
and lymph node, several mechanisms have been proposed leading to the damage of these organs. Mechanisms include are direct viral replication, cytokine storm, hypoxia, hypovolemia, acute on chronic injury of pre-existing comorbidities and drug related toxicity. The author stated that the autopsies show 2 patterns of injury, acute and chronic. Chronic pathologic changes include chronic renal disease namely diabetic nephropathy and vascular sclerosis, cardiac hypertrophy, patchy cardiac fibrosis, and coronary artery disease, as well as the hepatic steatosis. The acute changes include the pulmonary diffuse alveolar damage, widespread microscopic thrombi, reactive lymph node changes with immunoblastic- type cells, atypical hepatic sinusoidal cells, and acute tubular injury in the kidneys [2].

Schaller et al. [4] evaluated autopsies of 10 patients with COVID-19. It was found that changes in the respiratory tract was the leading cause of death in patients, showing acute and organizing diffuse alveolar damage on histopathological examination [4]. Carsana et al. [1] described the postmortem findings of 38 deceased patients, mainly focused on pulmonary lesions. Grossly, lungs appear to be heavy, oedematous and congested. The histopathological findings in lung tissue revealed exudative and early or intermediate proliferative phases of diffuse alveolar damage in all cases with focal features of interstitial, organizing, or acute fibrinous organizing pneumonia. Diffuse fibrin and platelet clots in the lumen of the peripheral small vessels of the lung was seen in 33/38 cases. It was also stated that there was reactive atypia in pneumocytes and diffuse peripheral small vessel thrombosis.

Several studies also demonstrated the similar findings in lung. They described that in lung injury, diffuse alveolar damage, including the exudative and proliferative phases are the common finding Also, inflammatory infiltrate, oedema, pneumocyte hyperplasia, fibrinous exudate, and organization were found [5,6]. Cardiovascular system was also frequently involved. Myocardial injury results from ischemia, heart failure, pulmonary embolism, tachycardia, and sepsis which is associated with elevated troponin levels. Lindner et al. [7]; Jacobs et al. [8] and Halushka et al. [9] published the findings seen in heart. Jacobs et al. [8] have studied endomyocardial biopsies 5 out of 104 patients with suspected myocarditis or unexplained heart failure. Jacobs et al. [8] and Lindner et al. [7] performed RTPCR and in situ hybridization, respectively on heart tissue. It was found out that the viral genome is present in myocardial tissue also in the interstitial cells or macrophages invading the myocardial tissue $[7,8]$. Mainly, lymphocytic myocarditis was seen in COVID19 patients. These lymphocytes are CD3-positive, T-lymphocytic which infiltrate the myocytes and is associated with myocyte damage. Myocardial inflammation caused by COVID19 was mainly mediated by macrophages and also by T-lymphocytes [8].

Halushka et al. [9] reported cardiac findings as non-myocarditis inflammatory infiltrate, single cell ischemia, acute myocardial infarctions and myocarditis. Myocarditis is defined as the presence of an inflammatory infiltrate with adjacent myocyte injury. In viral infections, the infiltrate is of lymphocytic predominant. Also, occasionally the predominant cell type in myocarditis can be eosinophils or rarely neutrophils. Myocarditis itself is sufficient to cause cardiac dysfunction, which can be diffuse or multifocal with a significant amount of infiltrate and myocyte injury.

Autopsy findings play an important role to study the pathophysiology of COVID19. But due to health hazards, they are being performed in a very limited number. The major findings on autopsies are diffuse alveolar damage with changes of pneumonia, myocarditis and formation of microthrombi due to endothelial dysfunction. Due to high propensity of thrombus formation, use of anticoagulants has been advised in COVID-19 patients.

\section{CONCLUSION}

Due to biosafety concerns, only limited number of medical autopsies was conducted. For the proper treatment of the patients, there is still insufficient data on pathophysiological of the coronavirus disease.

\section{ACKNOWLEDGEMENT}

All the authors have contributed to concept, data analysis, manuscript editing and review. There was no prior abstract/ poster presentation and publications related to this topic.

\section{REFERENCES}

1. Carsana L, Sonzogni A, Nasr A, Rossi RS, Pellegrinelli A, et al. (2020) Pulmonary post-mortem findings in a series of COVID-19 cases from northern Italy: A two-centre descriptive study. Lancet Infect Dis 20(10): $1135-140$

2. Elsoukkary SS, Mostyka M, Dillard A, Berman DR, Ma LX, et al. (2021) Autopsy findings in 32 patients with COVID-19: A single-institution experience. Pathobiology 88: 56-68.

3. Maiese A, Manetti AC, La Russa R (2021) Autopsy findings in COVID-19related deaths: a literature review. Forensic Sci Med Pathol 17(2): 279296.

4. Schaller T, Hirschbühl K, Burkhardt K (2020) Postmortem Examination of Patients With COVID-19. JAMA 323(24): 2518-2520.

5. Franks T, Chong P, Chui P (2003) Lung pathology of severe acute respiratory syndrome (SARS): A study of 8 autopsy cases from Singapore. Hum Pathol 34: 743-748.

6. Hwang DM, Chamberlain DW, Poutanen SM, Low DE, Asa SL, et al. (2005) Pulmonary pathology of severe acute respiratory syndrome in Toronto. Mod Pathol 18: 1-10.

7. Lindner D, Fitzek A, Bräuninger H, Aleshcheva G, Edler C, et al. (2020) Association of Cardiac Infection With SARS-CoV-2 in Confirmed COVID-19 Autopsy Cases. JAMA Cardiol 5(11): 1281-1285.

8. Jacobs W, Lammens M, Kerckhofs A, Voets E, San EV, et al. (2020) Fatal lymphocytic cardiac damage in coronavirus disease 2019 (COVID-19): autopsy reveals a ferroptosis signature. ESC Heart Failure 7: 3772-3781.

9. Halushka MK, Vander Heide RS (2021) Myocarditis is rare in COVID-19 autopsies: cardiovascular findings across 277 postmortem examinations. Cardiovasc Pathol 50:107300. 